rumson

glyndîn

Glyndŵr University

Glyndŵr University Research Online

Computing

Computer Science

$1-1-2007$

\title{
An argument for simple embedded ACL optimisation
}

Vic Grout

Glyndwr University, v.grout@glyndwr.ac.uk

John N. Davies

Glyndwr University, j.n.davies@glyndwr.ac.uk

John McGinn

Glyndwr University, j.mcginn@glyndwr.ac.uk

Follow this and additional works at: http://epubs.glyndwr.ac.uk/cair

Part of the Computer Engineering Commons

\section{Recommended Citation}

Grout, V., Davies, J., \& McGinn, J. (2007) 'An argument for simple embedded ACL optimisation'. Computer Communications, 30(2), 280-287

This Article is brought to you for free and open access by the Computer Science at Glyndŵr University Research Online. It has been accepted for inclusion in Computing by an authorized administrator of Glyndŵr University Research Online. For more information, please contact

d.jepson@glyndwr.ac.uk. 


\title{
An argument for simple embedded ACL optimisation
}

\begin{abstract}
The difficulty of efficiently reordering the rules in an Access Control List is considered and the essential optimisation problem formulated. The complexity of exact and sophisticated heuristics is noted along with their unsuitability for real time implementation embedded in the hardware of the network device. A simple alternative is proposed, in which a very limited rule reordering is considered following the processing of each packet. Simulation results are given from a range of traffic types. The method is shown to achieve savings that make its use worthwhile for lists longer than a given number of rules. This number is dependent on traffic characteristics but generally around 25 for typical network conditions.
\end{abstract}

\section{Keywords}

Access Control Lists, ACLs, Packet latency, Optimisation

\section{Disciplines}

Computer Engineering

Comments

Original publication is available at www.sciencedirect.com Copyright (C) 2006 Elsevier B.V. 


\title{
AN ARgument FOR Simple EMBEDDED ACL OPTIMISATION
}

\author{
Vic Grout ${ }^{1}$, John Davies and John McGinn \\ Centre for Applied Internet Research (CAIR) \\ University of Wales, NEWI, Wrexham, LL11 2AW, UK
}

\begin{abstract}
The difficulty of efficiently reordering the rules in an Access Control List is considered and the essential optimisation problem formulated. The complexity of exact and sophisticated heuristics is noted along with their unsuitability for real time implementation embedded in the hardware of the network device. A simple alternative is proposed, in which a very limited rule reordering is considered following the processing of each packet. Simulation results are given from a range of traffic types. The method is shown to achieve savings that make its use worthwhile for lists longer than a given number of rules. This number is dependent on traffic characteristics but generally around 25 for typical network conditions.
\end{abstract}

\section{Keywords Access Control Lists, ACLs, Packet latency, Optimisation}

\section{Introduction}

Access Control Lists (ACLs) are ubiquitous in internetworking. As the name suggests, they play a major role in the process of passing or blocking traffic through sensitive regions of a network. They can permit or deny traffic from or to given sources or destinations, or discriminate on the basis of content or other characteristics. As an extension to these simple security aspects of ACLs, certain traffic may be chosen for tunnelling in a Virtual Private Network $(V P N)$ for example.

However, this ability to filter network traffic makes ACLs suitable for a wider purpose. They may be applied in any situation in which there is a need to choose certain data packets for a given traffic policy. Network Address Translation (NAT), traffic shaping, various aspects of internet routing, and numerous other traffic policies, all require packets to which the policy is to be applied to be separated from those to which it is not. ACLs may vary considerably in size but it is not uncommon for a single packet to be tested against several ACLs on its passage across a single internet router and many more across a complete domain.

This paper considers the delay this process adds to the packet's progress. It discusses optimisation of ACL structure to make the process more efficient and thus reduce the

\footnotetext{
${ }^{1}$ Corresponding author - Tel. +44 (0)1978 293203 Fax. +44 (0)1978 293168
} 
delay. Optimisation is often a complex process, however. There is no value in an optimisation regime that takes longer to implement than the latency it saves, and this has often blocked attempts to implement ACL optimisation in real time and embedded within the interface (say) hardware of the router in all but the largest models. However, this paper demonstrates that a simple optimisation algorithm can be applied in this environment with reductions in packet latency that exceed the time taken for it to run. The essence of the technique is to consider a very limited reordering of the list of rules following the processing of every packet.

\section{The Problem}

An ACL is an ordered list of rules. Each rule accepts or rejects a packet based on one or some of its characteristic(s) - its profile. Typically, a packet may be considered on the basis of its source, destination or traffic type, although other features may be relevant [1]. Figure 1 gives an example of a typical ACL in the syntax of the Cisco Internetwork Operating System (IOS) [2]. The use of the terms permit and deny reflect the original role of ACLs in passing or blocking traffic.

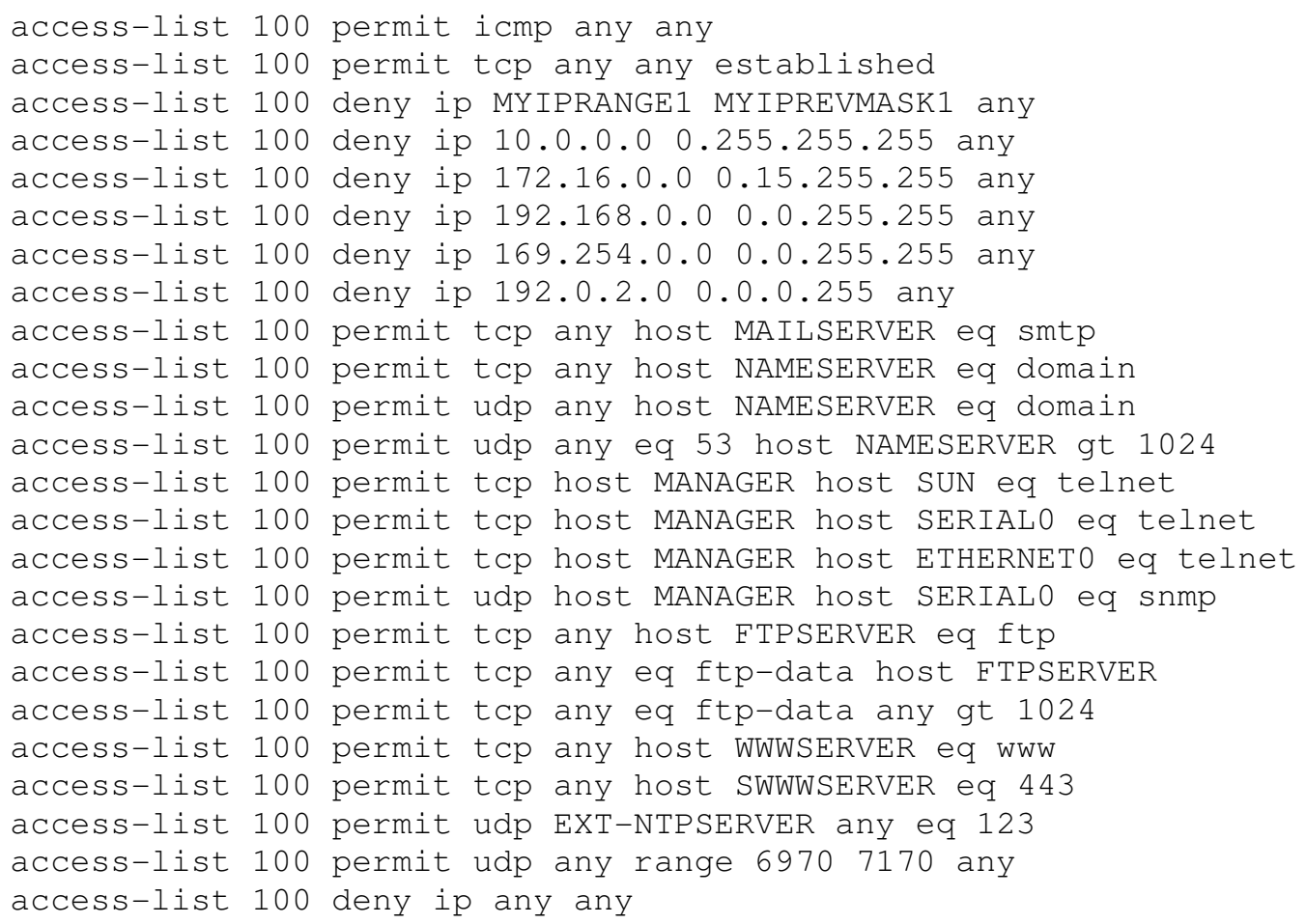

Figure 1. An Example of an Access Control List (ACL).

Each packet to be tested against an ACL is compared with the first rule, then the second, and so on, until a rule matches its profile. The rule is then permitted or denied accordingly and no more rules are considered. There is usually an implicit 'deny all' rule 
terminating each list to deal with packets not matched by any other rule. A precise treatment of rule and packet formats and profiles is given in [3]. This level of analysis is not required here except in its final formulation of the problem. However it is necessary to note that rule order is critical in an ACL ...

Consider two rules as follows: rule 1 permits packets with characteristic $A$ (source address, for example) and rule 2 denies packets with characteristic $B$ (destination address, say). A packet with a profile matching both characteristics (from $A$ to $B$ in this case) will match both rules. Consequently, the order of rule $1 \ldots$ rule 2 will permit the packet whereas the order rule 2 ... rule 1 will deny it. Not all rules will be dependent in this way but those that are must have their relative order in the list preserved if the ACL is to retain its intended purpose. Of course, this only applies for rules of opposite types. Several 'permit' rules in a contiguous block, for example, can be freely reordered among themselves.

Some rules will take longer to process than others and some are more likely to match packets than others. The difference in processing time comes from the level or extent to which a rule has to examine a packet, and the likelihood of a match, its hit-rate, will vary with changing traffic flows. For any given ACL, there may be a better version, with rules in a different order, which performs the same task more efficiently - always remembering that any reordering must preserve the order of dependent rules.

For straightforwardness in what follows, we denote the rule at position $i$ in an ACL simply as rule $i$. Then for a given rule $i$, in an ACL $A$, define its latency, $l_{i}$, to be the time taken to match it against a single packet and its hit-rate, $h_{i}$, to be the probability that the next packet will match the rule, at its present location in $A$ (and not any rule $j<i$ that precedes it). Then the cumulative latency of rule $i, \lambda_{i}$, is given by

$$
\lambda_{i}=\sum_{j=1}^{i} l_{i},
$$

the sum of the latencies of all rules up to and including $i$. The expected latency of $A, E_{A}$, the average time taken to process the list, is then

$$
E_{A}=\sum_{i=1}^{n} h_{i} \lambda_{i}
$$

where there are $n$ rules in $A$. Define the dependency matrix, $D=\left(d_{i j}\right)$ as $d_{i j}=1$ if rules $i$ and $j$ are dependent in $A$ and $d_{i j}=0$ otherwise. Starting from an initial (probably administratively defined) ACL, $A_{0}$, the optimisation problem then is then to find the list, $A^{*}$, with minimum expected latency, obeying the dependency constraints, that is,

$$
E_{A^{*}}=\min _{A} E_{A}
$$


subject to the constraint that, for any rules $i$ and $j$ with $d_{i j}=1$ in $A_{0}$, their relative order (but not necessarily exact position) must be preserved in $A^{*}$.

We consider changing traffic profiles as the paper progresses. However, even in its static form, the problem is complex - NP-complete in fact [3]. No exact solutions are to be found in reasonable time on any platform.

The efficiency of ACL structure is first considered theoretically in [4] and [5]. Cisco [6] provide the first real attempt at optimisation. Their "Hits Optimizer" records which rules match packets in real time on the router, then their "ACL Optimizer" works offline to reorder the rules in line with dependency constraints. Apart from the obvious limitation of working offline, this system does not discriminate between rules of different latencies. Bukhatwa and Patel [7] demonstrate the savings available from ACL optimisation but ignore both rule dependencies and differing rule latencies. An improved approach [8] gives a simplified method for reordering rules based on latency but still ignores dependencies. Both methods are implemented offline. Cisco [9] introduce "Turbo Access Lists" with rules searched as look-up tables but only on high-end routers and specialist firewalls.

Al Shaer and Hamed [10] give an improved formulation of the problem for the purposes of detecting rule anomalies. An alternative for the full problem is given by Grout and McGinn [3] along with a simple, but not particularly efficient, method of solution that makes real-time online optimisation possible for moderate numbers of rules. The complexity of the algorithm is around $O\left(n^{3}\right)$ - impractical for large lists. Finally, Grout et al. [11] offer an efficient heuristic. However, at the time of going to print, the paper left some questions unanswered. It is the purpose of this paper to finish the story by completing the analysis, modifying the algorithm slightly and testing and establishing the value of key parameters.

\section{An Efficient Solution}

Grout et al. [11] note the following:

- In comparing rule order for a list $A$, the significance of rule hit-rates is only relative. It is not necessary for them to be normalised (i.e. summing to 1 ) probabilities. This implies that the hit-rate of a newly hit rule, $i$, can increase without changing the hitrates of the other rules.

- Following an increase in a rule $i$ 's hit-rate, the only possible change in rule order (to reduce $E_{A}$ ) is to promote $i$ up the list. The most likely candidate with which to exchange it is rule $i-1$, immediately above it.

- The potential saving in expected latency in swapping rules $i-1$ and $i$ is given by $h_{i} l_{i-1}-$ $h_{i-1} l_{i}$ (see the original paper for the full expansion), a simple, local calculation.

- Considering rule promotions continuously in this manner is entirely responsive to dynamically changing traffic patterns. 
These observations allow the search process to be simplified considerably. A simple three-part heuristic algorithm for ACL optimisation is then proposed as follows:

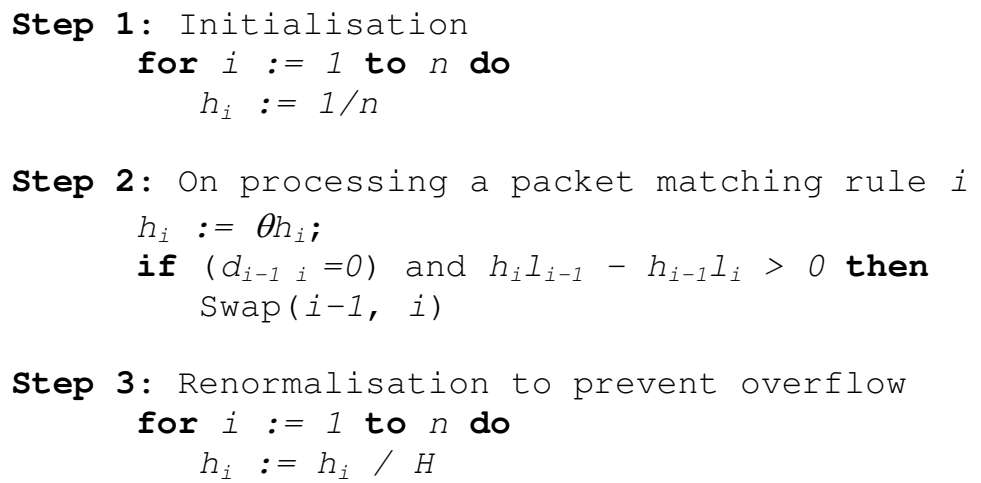

Step 1 sets all rule hit-rates to be equal, normalised probabilites when the ACL is initially configured (or reconfigured). Step 2 is executed after the processing of every packet. The hit-rate of the matched rule is increased by a factor $\theta$, the promotion coefficient, and the rule is swapped with its predecessor if the two are independent and the hitrate/latency trade off is favourable. (Note, for the analysis to follow, that swapping two rules also entails swapping their respective hit-rates.) At certain intervals, Step 3 stops the hit-rates increasing without bound and thus prevents overflow. In the original paper, $H$ is the sum of the individual hit-rates for all rules, $H=\sum_{i=1}^{n} h_{i}$, thus renormalising values. Steps 1 and 3, of order $O(n)$ are executed infrequently whilst the continuously used Step 2 is a single simple calculation. The algorithm places an exponentially decreasing importance on older rule matches, parameterised by $\theta$.

Grout et al. [11] leave the following questions largely unanswered with respect to this algorithm. What should the value of $\theta$ be? How frequently is Step 3 necessary?

Before continuing, this paper revises (streamlines) the above algorithm as follows:

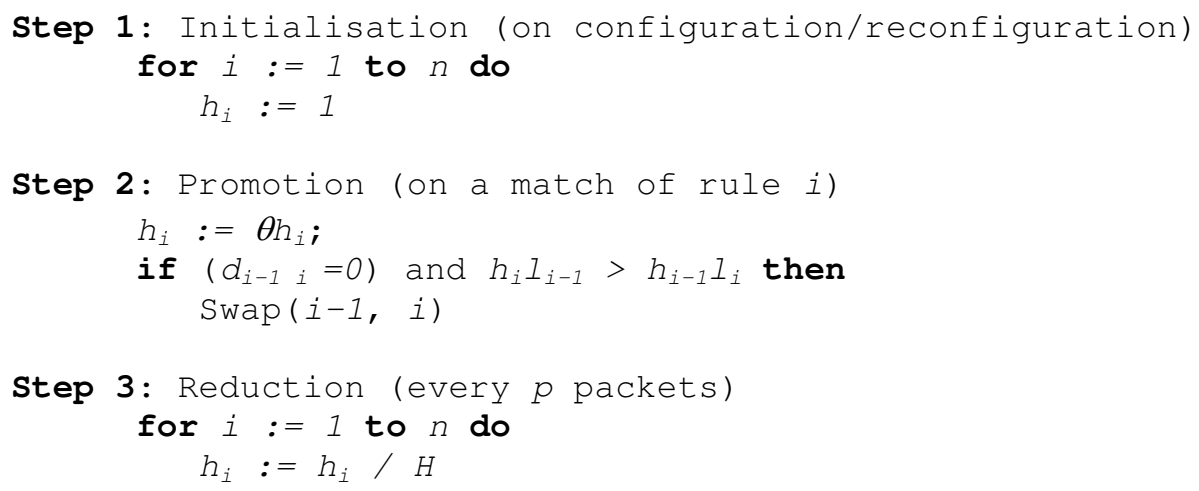

The simplified initialisation (Step 1) reflects the fact that the hit-rates need not be normalised. Step 2 remains trivial. 
$p$ and $H$ are easily determined. The fastest route to overflow is through a stream of packets all matching the same rule. The hit-rate of this rule will increase by a factor $\theta$ on each packet and, after $a$ packets, will have a hit rate of $\theta^{a}$. If $M$ is the largest value permitted in the data range being used, then $p$ can be calculated as $p=\left\lfloor\log _{\theta} M\right\rfloor$, a router constant. ( $\lfloor x\rfloor$ is the integer part of $x$.) Taking $H=\max _{i} h_{i}$ will reduce the maximum value back to 1 each time Step 3 is executed. It remains to determine the value for $\theta$.

\section{Simulation and Results}

The intention of this paper is to establish, in principle, the viability of a simple, embedded heuristic optimisation technique acting upon rule order in ACLs. This is fortunate because the process of obtaining results from live ACLs is hindered by a number of factors:

- Our initial aim is to be able to characterise both ACLs and traffic flows, and the relationship between them. ACLs are characterised by their size, the level of interdependence between rules and the distribution of rule hit-rates. (For some ACLs, hit-rates may be close to uniform and/or largely independent of rule order; in others, some rules - typically those close to the start - may have hit-rates considerably larger than the rest.) Traffic is characterised, in particular, by its stability: a stream of similar packets may match the same rule whereas unstable flows will find different matches throughout an ACL. Our ultimate aim is to investigate how appropriate our proposed method will be for a spectrum of ACL types and traffic flows. This level of parameterisation will be difficult to obtain from production lists and flows. Although there are some sources of 'real' traffic for simulation purposes, ACLs are harder to find: their data is simply not available in sufficient, measurable quantity (at least in their thousands) to enable us to produce consistent results.

- The low-level implementation of packet-matching techniques varies considerably from platform to platform [12]. Although primarily hardware-based, the precise relationship with the (eg, router) operating system is vendor-, and sometimes model-, dependent. Even in hardware alone, the number of steps to perform any operation will depend on the unique architecture and organisation of the platform. Using real ACLs for simulation serves little purpose if their performance varies between models. Using an abstract simulation with parameterisation that can be tailored to any given environment is actually a stronger proposition.

- Experimenting with the proposed algorithm, and testing variations of key parameters $(\mathrm{eg}, \theta)$ in a working environment is difficult on some platforms, particularly in hardware implementations. Without access to the embedded hardware itself, experimentation is impossible on most production routers. Limited control is possible on some platforms ([13] for example) but can only test a small proportion of cases. The final alternative is a general network simulator. ns-2, for example [14], offers some generic packet-processing functionality. However, such simulations are generally small and, abstracted as they themselves are, no more accurate or 'real' than the processes described below. 
Table I. Simulated Results: Rank and Cumulative Latencies (example).

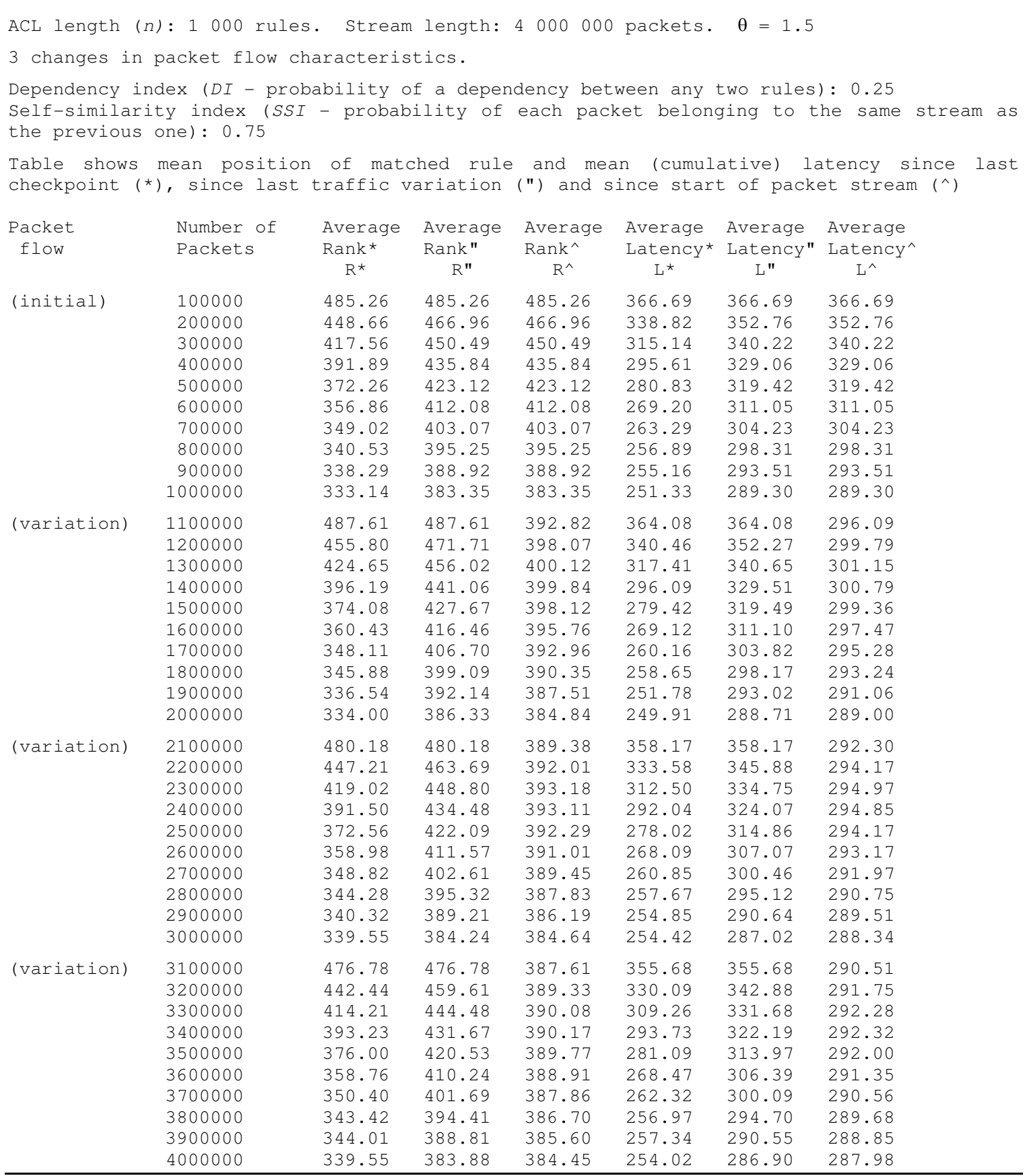

Our simulation is based on an in-house numerical model, capable of generating ACLs and traffic flows according to a given parameter set, described as follows. For tested ACLs, the number of rules $(n)$ ranged from 10 to 10000 . Dependencies between rules were determined using a dependency index, $D I$, the probability that any two rules are dependent. Values of $D I$ in the range $O$ (no dependencies) to 1 (complete dependency) were used. For each rule pair, $(i, j)$, dependencies are randomised as $d_{i j}=1$ with probability $D I$ and $O$ otherwise. Rule latencies were uniformly randomised from $0.5 \mu$ s to 1.0 s. Actual values depend on the router hardware of course [14] but it is only relative 
values that are significant. (Routers that process packets faster will also optimise faster see the conclusions section that follows.)

For traffic, the simulation is more sophisticated. The traffic simulator generates packets with given probabilities of matching each rule in the list. At intervals, these probabilities may change to reflect shifting traffic patterns. Within a single traffic pattern, however, there is a certain probability that a packet is identical to the previous one - or part of a similar stream - and matches the same rule.

So, at the start of the simulation, a value of the self-similarity index, SSI, is set. Then a match probability, $\rho_{i}$ is randomised for each rule $i$ and normalised so that $\sum_{i=1}^{n} \rho_{i}=1$. The first packet is generated, matching rule $i$ with probability $\rho_{i}$. Subsequent packets match the same rule with probability $S S I$, and otherwise match any rule according to the match probabilities, $\rho_{i}$. Every $q$ packets, the match probabilities, $\rho_{i}$, are re-randomised.

$n$ and $D I$ can be set to produce different types of ACL while $q$ and SSI vary to reflect different types of traffic. As an example, Table I records simulated output from a test with $\theta=1.5, n=1000, D I=0.25, q=1000000$ and $S S I=0.75 .4000000$ packets are generated in total, in four stages with varying profiles. Results are reported every 100000 packets.

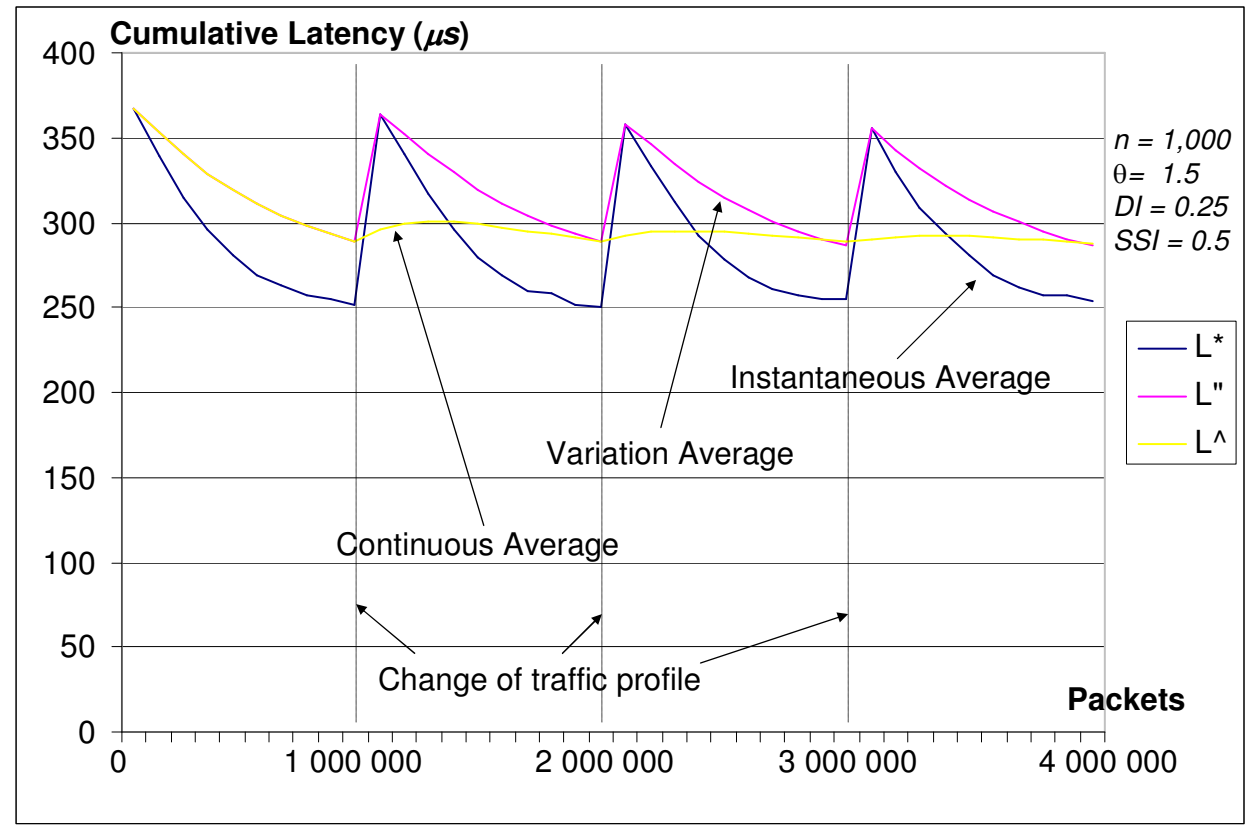

Figure 2. Simulated Results: Cumulative Latencies.

Tabled results are the mean position of the matched rule (rank) in the ACL and the mean cumulative latency of this rule. In both cases, three values are given: the mean since the last set of figures $\left(R^{*} \& L^{*}\right)$ - the instantaneous average, the mean since the last traffic variation $\left(R\right.$ ” \& $\left.L^{\prime \prime}\right)$ - the variation average, and the mean of the entire simulation $\left(R^{\wedge} \&\right.$ 
$\left.L^{\wedge}\right)$ - the continuous average. The three latency averages, $L^{*}, L^{\prime}$ and $L^{\wedge}$, are plotted in Figure 2 .

The mean rank, $R$, for a 1000 rule list with no optimisation will be 500 and the mean cumulative latency, $L$, for a latency range of 0.5 to $1.0,500 \times(1.0+0.5) / 2=375$. In simulation, optimised averages start at these values and are then progressively lowered as rules with high hit rates are promoted. When traffic profiles change, instantaneous and variation averages become poor again but are gradually improved once more as the ACL adapts to the new characteristics. The continuous average becomes steadier. In this example, $L^{\wedge}$ approaches a figure of approximately 287 , an improvement of $23 \%$ on the non-optimised figure.

Table II. Simulated Results: Traffic Parameters and Promotion Coefficient.

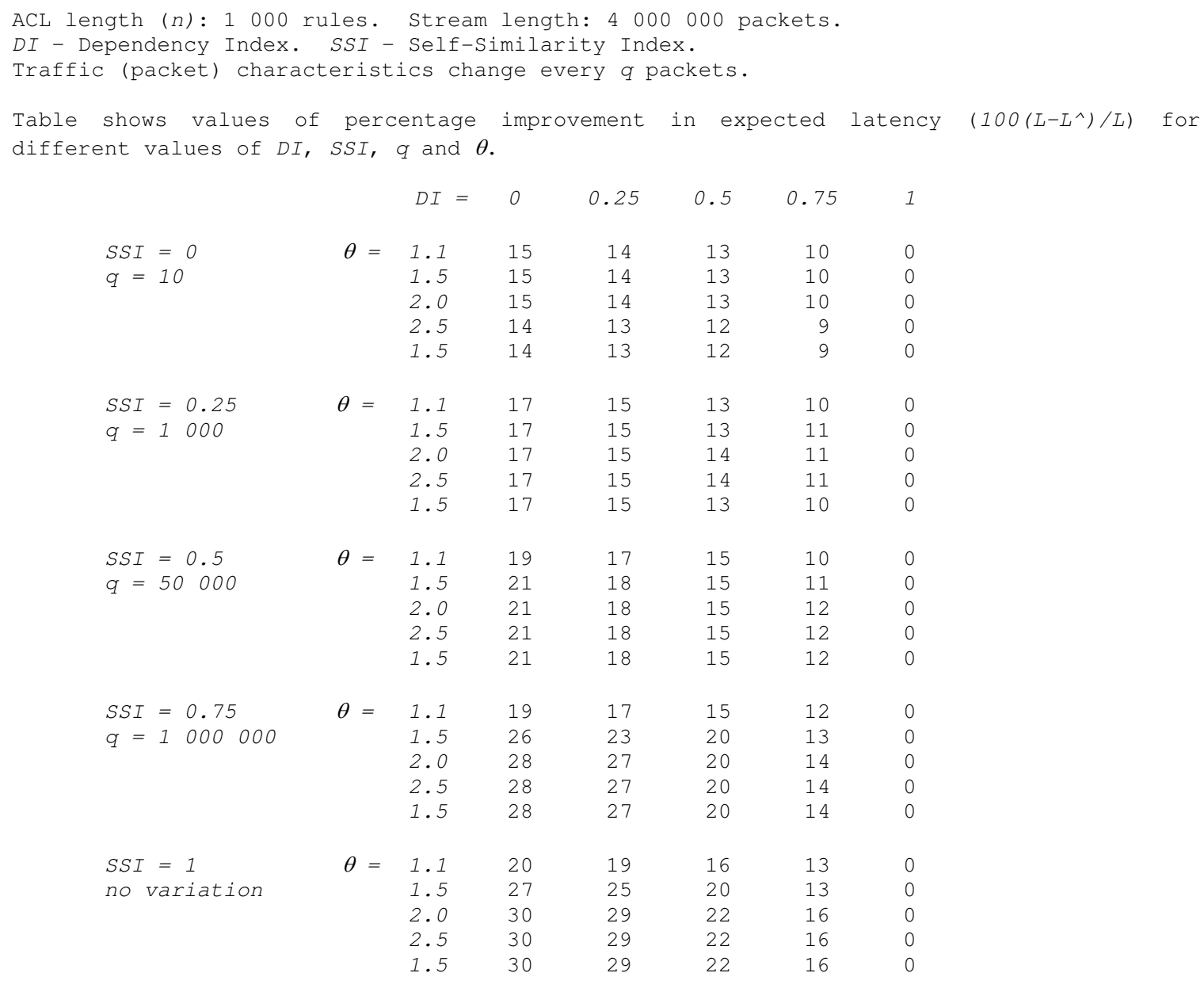

Different parameters affect these values as shown in Table II. Results are proportionally similar for different $n$. High values of $D I$ work against the optimisation process, prohibiting desirable swaps. In the extreme cases, $D I=1$ prevents any optimisation whereas $D I=0$ allows rules to move freely. High values of $q$ and SSI imply greater traffic stability, which improves the optimised values. The effect of $\theta$ is more subtle. 
High values make rule promotion faster, which works well for self-similar, stable traffic but can lead to repetitive, unnecessary swaps for continuously changing, or oscillating, traffic patterns. A balance is necessary: a value around $\theta=2$ maximises the improvement in expected latency in most cases.

\section{Analysis}

Routers vary considerably in their operation, particularly in terms of implementation in hardware. The following is, by necessity, generic and, to some extent, imprecise. However, it gives an appropriate indication of the relative worth of dynamic optimisation. We discuss an operation simply as a unit of calculation or assignment, probably performed in hardware on the appropriate interface. (However, the same argument would apply in relative terms if these operations were to be a part of the operating system software.)

With a $B$-bit data type/range (e.g. register size) and $\theta=2$, we have $p=\left\lfloor\log _{2} 2^{B}\right\rfloor=B$. For any given ACL manual configuration (or reconfiguration), Step 1 of the algorithm is executed once and can be taken as part of the configuration, Step 2, every processed packet and Step 3 every $B$ packets. Step 2 consists of an assignment, two calculations, two comparisons and a conjunction (possibly) followed by a swap of six assignments three for the rules and three for their hit-rates - twelve operations in all. Step 3 has two loops of size $n$, one to establish the maximum value and the other to reduce each value. The mean complexity (of Step 3) each packet is then $2 n / B$ and, in total, $12+2 n / B$ for Steps 2 \& 3 combined.

Table III. Optimisation Trade-Off - Saving against Cost

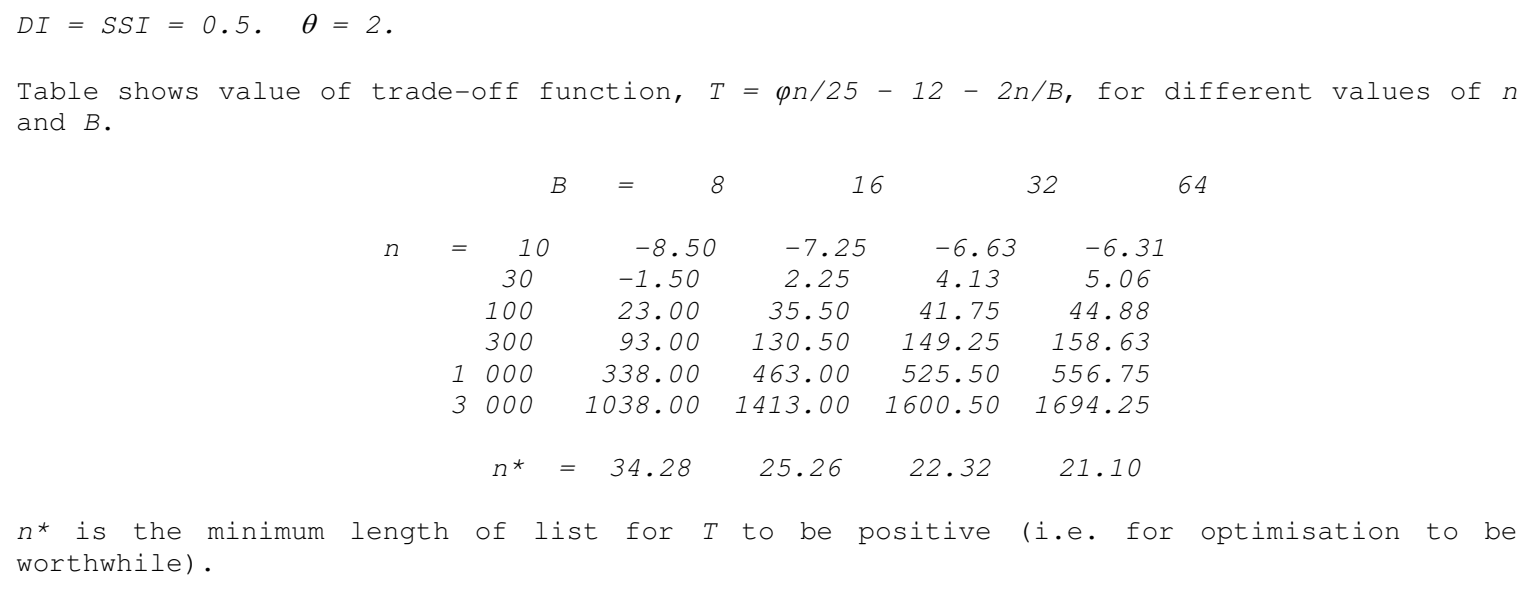

Matching a packet against a rule consists of at least one operation (permit or deny) followed by between 1 and 5 comparisons (Figure 1). Taking a mean of $1+3=4$ operations per rule and a percentage saving for an optimised list of $\varphi$ gives an optimisation trade-off of 


$$
T=\frac{4 \varphi n}{100}-12-\frac{2 n}{B}
$$

which will be positive (i.e. worthwhile) when

$$
\varphi>\frac{300}{n}+\frac{50}{B}
$$

For example, taking $n=1000$ and $B=16$, this gives $300 / 1000+50 / 16=3.425$. Table II shows that the improvement, $\varphi$, exceeds this for all values other than $D I=1$ and is therefore worthwhile. Alternatively, taking $\theta=2$ and $D I=S S I=0.5$ gives an improvement of $\varphi=15$ and a trade-off of $T=(15 \times 1000) / 25-12-2000 / 16=463$, a positive benefit. Table III extends this calculation across a range of values of $n$ and $B$ and, for each $B$, shows the key value of $n^{*}$, the size of ACL for which optimisation is profitable. Table IV fixes $B$ at 16 and calculates $n^{*}$ for various values of DI and SSI.

Table IV. Optimisation Trade-Off - Minimum ACL Length

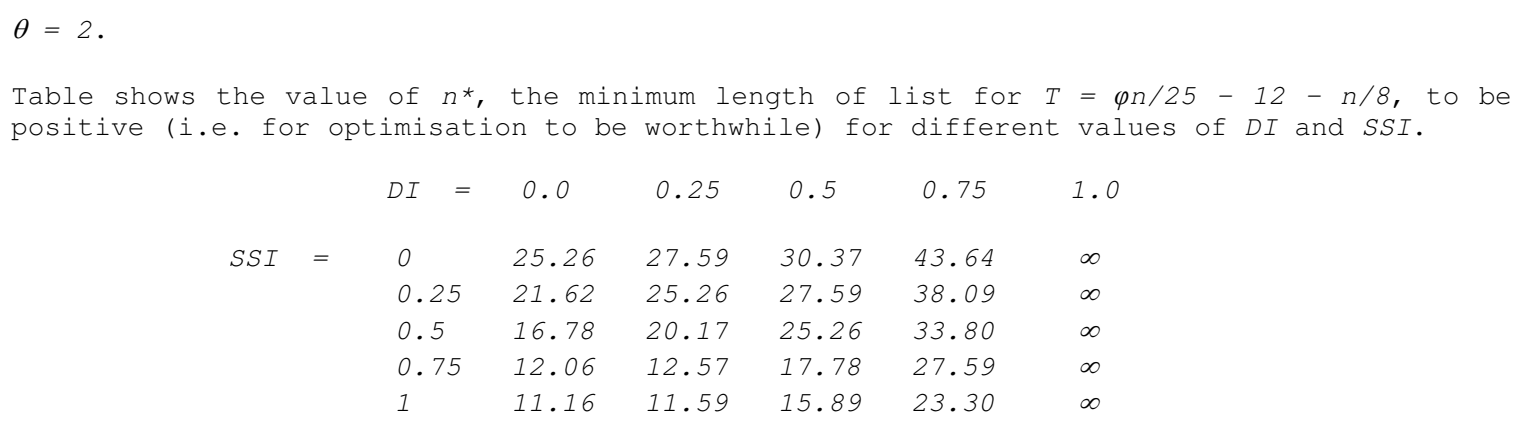

\section{Conclusions}

No amount of traffic modelling can substitute entirely for testing on production routers. However, our simulations are extensive and, within themselves, give consistent results.

The major obstacle to successful (worthwhile) optimisation is highly interdependent rules in an ACL. If no or few rules are permitted to be reordered then it is impossible or difficult to find equivalent lists with lower expected latencies. However, this is rarely the case in practical ACLs. The typical ACL in Figure 1, for example, has large blocks of separate 'permit' and 'deny' blocks with no dependencies within them. A worst-case figure for a practical ACL is likely to be $D I \approx 0.5$, giving good results (Tables II \& IV).

Table II suggests $\theta=2$ as an appropriate (and, in fact, convenient) value for the promotion coefficient. The number of packets between hit-rate reductions (Step 3) is then $B$, the size (number of bits) of the register being used to store them. The final version of the three part algorithm then becomes: 


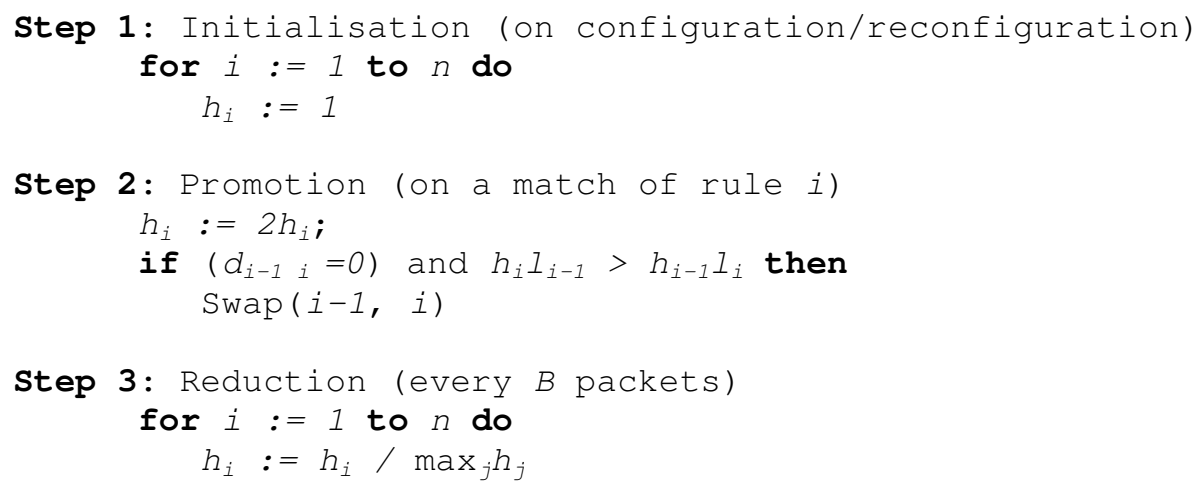

Depending on the stability and self-similarity of the traffic ( $q$ and $S S I$ ) and the frequency of hit-rate reduction $(B)$, optimisation becomes worthwhile for ACLs above a certain length $\left(n^{*}\right)$ (Tables III \& IV). For realistic dependencies, this figure ranges between about 10 and 30 . It will be trivial to separate those lists to which optimisation is to be applied from those to which it is not. Of course, it is precisely for longer ACLs that optimisation will yield the best results.

Real-time, online, embedded ACL optimisation on operational routers, as proposed, is possible and worthwhile. It is now recommended that it be put into practice as an integral part of the router hardware for practical testing.

\section{References}

[1] Access Control Lists, Cisco Systems, USA,

(http://www.cisco.com/univercd/cc/td/doc/product/software/ios113ed/113ed_cr/secu r_c/scprt3/scacls.htm)

[2] JANET-CERT Example Router Configuration, (http://www.ja. net/CERT/JANETCERT/prevention/template.html)

[3] Grout, V. and McGinn, J., Optimisation of Policy-Based Routing Using Access Control Lists, IFIP/IEEE Symposium on Integrated Network Management, Nice, France, $16^{\text {th }}-19^{\text {th }}$ May 2005

(full version available at http://www. newi.ac.uk/groutv/papers/acls.pdf)

[4] Hari, B., Suri, S. and Parulkar, G., Detecting and Resolving Packet Filter Conflicts, Proceedings of the $19^{\text {th }}$ Joint Conference of the IEEE Computer and Communications Societies (INFOCOMOO), pp1203-1212, 2000.

[5] Stoica, I., Route Lookup and Packet Classification, Technical Report No. CS 268, Department of Electrical Engineering and Computer Science, University of California, Berkeley, USA, 2001.

[6] ACL Optimizer and Hits Optimizer, Cisco Systems, USA, (http://www.cisco.com/univercd/cc/td/doc/product/rtrmgmt/cw2000/fam_prod/acl_mgr /aclm_1_x/1_6/u_guide/ac1js.htm) 
[7] Bukhatwa, F. and Patel, A., Effects of Ordered Access Lists in Firewalls, Proceedings of IADIS WWW/Internet International Conference (W3I 2004), Algarve, Portugal, $5^{\text {th }}-8^{\text {th }}$

November 2003, pp257-264.

[8] Bukhatwa, F., High Cost Elimination Method for Best Class Permutation in Access Lists, Proceedings of IADIS WWW/Internet International Conference (W3I 2003), Madrid, Spain, $6^{\text {th }}-$ $9^{\text {th }}$ October 2004, pp287-294.

[9] Turbo Access Control Lists, Cisco Systems, USA,

(http://www.cisco.com/univercd/cc/td/doc/product/software/ios121/121newft/121t/1 21 t5/dttacl.htm)

[10] Al-Shaer, E. and Hamed, H., Modeling and Management of Firewall Policies, IEEE Transactions on Network and Service Management, Vol. 1-1, April 2004.

[11] Grout, V., McGinn, J. and Davies, J., Reducing Processing Latency in Network Packet Filters, Proceedings of the Fifth International Network Conference (INC 2005), Samos, Greece, July 2005, pp3-10.

[12] Varghese, G., Networking Algorithmics: An interdisciplinary approach to designing fast networking devices, Morgan Kaufmann, 2005.

[14] The Network Simulator - ns-2,

(http://www.isi.edu/nsnam/ns/)

[15] Suehring, S. and Ziegler, R., Linux Firewalls ( $3^{\text {rd }}$ edition), Novell Press, 2005.

\section{Biographies}

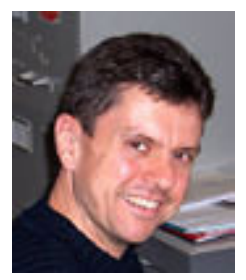

Vic Grout was awarded the BSc(Hons) degree in Mathematics and Computing from the University of Exeter (UK) in 1984 and the $\mathrm{PhD}$ degree in Communication Engineering from Plymouth Polytechnic (UK) in 1988.

He has worked in senior positions in both academia and industry for twenty years and has published and presented over 100 research papers. He is currently a Reader in Computer Science at the University of Wales NEWI, Wrexham in the UK, where he leads the Centre for Applied Internet Research (CAIR). His research interests and those of his research students span several areas of computational mathematics, particularly the application of heuristic principles to large-scale problems in network design and management.

Dr. Grout is a Chartered Engineer, Scientist and Mathematician and a Fellow of the British Computer Society (BCS). He chairs the biennial international conference series on Internet Technologies and Applications (ITA).

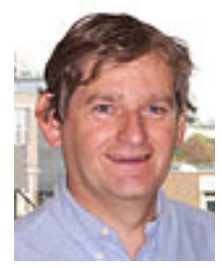

John Davies has a BSc(Hons) in Control Engineering from the University of Salford, UK (1973). He has worked for British Nuclear Fuels, Sension, the University of London Computer Centre and Daresbury Laboratories (UK) as a Project Manager, Chief Engineer, Senior Lecturer and Higher Scientific Officer respectively. He is currently a Senior Lecturer in Computing at the University of Wales, NEWI (UK) completing a $\mathrm{PhD}$ in network traffic prediction.

John has research interests in various aspects of network measurement, simulation and management and has published a number of technical papers on network routing, traffic congestion and optimisation. He is a member of the Institution of Engineering and Technology (IET) 


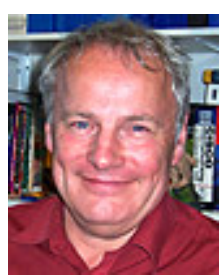

John McGinn was awarded the BSc(Hons) degree in Multimedia Computing by the University of Wales in 2000 and is currently working towards the $\mathrm{PhD}$ degree as a Research Fellow in the Centre for Applied Internet Research (CAIR) at the University of Wales, NEWI (UK).

John's research interests include network protocols and standards and distributed collaboration and visualisation. He has published and presented a number of technical papers on topics from information visualisation to ACL optimisation. He is a member of the British Computer Society (BCS) and the Institution of Engineering and Technology (IET). 